\title{
Multi-objective robust resource allocation for secure communication in full-duplex MIMO systems
}

\author{
Min Zhu ${ }^{1,2}$ and Dengyin Zhang ${ }^{1 *}$
}

\begin{abstract}
In this paper, we study robust resource allocation for the multi-user full-duplex (FD) multiple-input multiple-output (MIMO) communication systems. Particularly, we aim at minimizing uplink (UL) transmit power and downlink (DL) transmit power simultaneously while guaranteeing the quality of service (QoS) requirements regarding secure UL and DL communication, under the consideration of the imperfect channel state information (CSI) of the wiretap channels and the inter-user interference channels. In view of the conflicting of two objectives, we propose a multi-objective optimization (MOO) framework to achieve the trade-off between them. The formulated MOO problem is non-convex and intractable. By employing the weighted Tchebycheff, the Taylor series expansion, and the S-procedure approaches, we convert the MOO problem into the convex one and propose an iterative algorithm to solve it optimally. Simulation results not only demonstrate an interesting trade-off between the considered conflicting objectives but also show the efficiency of our proposed robust resource allocation designs.
\end{abstract}

Keywords: Robust secrecy beamforming, Wireless information and power transfer, Multi-input single-output (MISO), Physical layer security

\section{Introduction}

Owing to the exponential growth of demand in ubiquitous high data rate and secure wireless communication, the next generation communication systems suffer from severe limitation in radio resources such as energy and bandwidth. A promising technique for reducing the energy consumption and improving the bandwidth efficiency is multiple-input multiple-output (MIMO) which guarantees the efficient radio resource allocation by providing the extra degree of freedom. Moreover, as a practical realization, the multi-user MIMO (MU-MIMO) wireless communication systems which possess multiple receivers and shift the computational complexity from the receivers to the multi-antenna base station (BS) have drawn much attention of the researchers [1-5]. However, though the employment of the MU-MIMO wireless communication systems facilitate the efficiency for radio

\footnotetext{
*Correspondence: zhangdy@njupt.edu.cn

${ }^{1}$ Internet of Things, Nanjing University of Posts and Telecommunications, Nanjing 210003, China

Full list of author information is available at the end of the article
}

resource allocation, the spectral resource is still underutilized since the uplink (UL) and downlink (DL) transmission phases must be operated orthogonally in either time or frequency under the regime of half-duplex (HD) BS.

Recently, full-duplex (FD) wireless communication systems attract significant attention from both the academia and industry due to the ability in supporting the concurrent transmission and reception over the same frequency, which, thus, double the spectral efficiency compared with the HD communication systems [5-8]. However, owing to the strong loopback self-interference (SI), the implementation of FD communication encounters big challenges in the past decades. The protocols and resource allocation schemes need to be redesigned to fit with the context of FD communications. In [5], the authors studied the dynamic resource allocation and scheduling for the FD and hybrid relaying MIMO orthogonal frequency division multiple access (MIMO-OFDMA) systems. The authors in [6] studied the low-complexity scheme for the end-toend performance in MIMO FD relay systems under the consideration of outage. A close to optimal beamformer

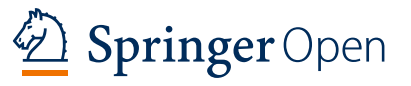

(c) The Author(s). 2017 Open Access This article is distributed under the terms of the Creative Commons Attribution 4.0 International License (http://creativecommons.org/licenses/by/4.0/), which permits unrestricted use, distribution, and reproduction in any medium, provided you give appropriate credit to the original author(s) and the source, provide a link to the Creative Commons license, and indicate if changes were made. 
design is proposed in [7] to maximize the spectral efficiency of FD small cell wireless systems. In [8], to restrain the SI and promote the spectral efficiency, both the massive MIMO antenna array and the multi-pair FD relays are introduced. Nevertheless, resource allocation scheme design for the systems with simultaneous UL and DL wireless communications have not been discussed in above literatures yet. Moreover, the UL and DL transmit power minimization are two conflicting design objectives. Thus, the frameworks adopted for the single-objective optimization in [5-8] may not be applicable in this scenario.

Simultaneous UL and DL wireless communication in FD systems have been studied in many literatures, recently. In [9], the authors investigated the robust power efficient problems under the premise of secure communication for the UL and DL users with FD BS. A multi-objective optimization framework was proposed to balance different design indexes. The similar system was considered in [10], where, in addition, the dual use of the radio frequency (RF) signals for simultaneous wireless information and power transfer (SWIPT) are incorporated. In [11], the multi-objective resource allocation problem is studied for multi-user multiple-input single-output (MISO) secondary communication system with SWIPT. By exploiting both the primal and dual solutions of the semidefinite relaxation (SDR) problem, the global optimal solution of the original problem is constructed. However, the problems investigated in above literatures are mainly focused on the MISO systems. The more complex systems, MIMO systems, are still remains little research, especially for the FD systems with simultaneous UL and DL transmission.

Motivated by the aforementioned observations, in this paper, we study the UL and DL transmission power minimization problem for the multi-user full-duplex (FD) multiple-input multiple-output (MIMO) communication systems. From the perspective of practice, the problem formulation takes into account the imperfectness of the CSI of the wiretap channels and the interference channels between UL user and DL users. Based on the MOO framework, the considered non-convex optimization problem is resolved by employing the weighted Tchebycheff, the Taylor series expansion, and the extended S-procedure approaches. Finally, from the derived approximate optimal solutions, the robust resource allocation schemes which balance UL and DL power consumption are proposed.

Notation: The lower-case, boldface lowercase, and boldface upper-case letters are used to denote scalars, vectors, and matrices, respectively; $\operatorname{Tr}(\mathbf{X}),|\mathbf{X}|(\operatorname{det}(\mathbf{X})), \mathbf{X}^{H},(\mathbf{X})^{-1}$, and $\|\mathbf{X}\|_{F}$ denote trace, determinant, Hermitian transpose, inverse, and Frobenius norm; I represents the identity matrix; $C^{n \times m}$ denotes the space of $n \times m$ complex matrices; $\mathbf{x} \sim C N(\boldsymbol{\mu}, \boldsymbol{\Sigma})$ means that the random vector $\mathbf{x}$ follows a circularly symmetric complex Gaussian (CSCG) distribution with mean $\boldsymbol{\mu}$ and covariance $\boldsymbol{\Sigma} ; \mathbf{X} \geq \mathbf{0}(\mathbf{X}>\mathbf{0})$ means that $\mathbf{X}$ is positive semidefinite (definite); $\mathbf{H}_{+}^{n}$ represents $n \times n$ Hermitian positive semidefinite matrices; $\mathbb{R}^{n}$ means $\mathrm{n}$-dimensional real vectors; $\operatorname{vec}(\mathbf{X})$ denotes the vectorization of matrix of $\mathbf{X}$ by stacking its columns; $\mathbf{X} \otimes \mathbf{Y}$ denotes the Kronecker product of $\mathbf{X}$ and $\mathbf{Y}$. Diag $(\mathbf{X})$ returns a diagonal matrix containing the diagonal elements of matrix $\mathbf{X}$ in its main diagonal.

\section{System model}

We consider a multi-user FD MIMO communication system which is comprised of an FD radio base station (BS), an UL user, $K$ DL users, and $M$ eavesdroppers. The BS is assumed to be equipped with $N_{B}>1$ antennas while the $k$ th DL user and $m$ th eavesdropper are equipped with $N_{D}>1$ and $N_{E}>1$ antennas, respectively. To facilitate efficient UL information transfer, which may be wiretapped by the eavesdroppers, we also presume that the UL user is equipped with multiple, $N_{U}>1$, antennas. Considering the existence of the eavesdroppers in our system, for guaranteeing the secure communication of both UL and DL channels, an efficient resource allocation scheme by joint design of UL/DL transmit covariance matrices has to be employed. The potential applications of our considered system could be the cognitive radio networks with full-duplex primary transmitter who simultaneous receives the confidential messages sent by the secondary transmitter and transmits confidential signals to the desired primary users via the same spectrum.

Remark 1 Note that for simplicity, in this paper, we consider the multi-user full-duplex MIMO communication system with only one uplink user. The more general multiple uplink users setup will be left as our future work. The single uplink user setup is widely assumed in the literatures for mathematical tractability and optimization [12]. Moreover, it is also of practical interest in many scenarios due to its low implementation complexity. For example, for the single uplink user setup, there is no multiple access interference, while for the multiple uplink users setup, the complicated multiple access interference management is required to suppress the co-channel interference among the uplink users $[9,10]$.

For the UL communication, the UL user transmits signal vector $\mathbf{u}(t) \in \mathbb{C}^{N_{U}}$, which is modeled as a complex Gaussian random vector with $\mathbf{u}(t) \sim C N(\mathbf{0}, \mathbf{\Sigma})$, where $\boldsymbol{\Sigma} \succeq \mathbf{0}\left(\Sigma \in \mathbf{H}_{+}^{N_{U}}\right)$ is the transmit covariance and is to be designed, to the FD BS. For the DL communication, sharing the same frequency with the UL channel, the FD BS transmits K independent signal streams to the K DL users. Meanwhile, the artificial noise (AN) is also generated by the FD BS to disturb the reception of the eavesdroppers. 
Thus, the transmitted signal at the FD BS possesses the following form

$$
\mathbf{x}(t)=\sum_{k=1}^{K} \mathbf{s}_{k}(t)+\mathbf{z}(t)
$$

where $k \in K, K \triangleq\{1, \cdots, K\} ; \mathbf{s}_{k}(t) \in \mathbb{C}^{N_{B}}$ denotes the confidential signal intend for the $k$ th DL user, which follows a complex Gaussian distribution $C N\left(\mathbf{0}, \mathbf{W}_{k}\right)$, $\mathbf{W}_{k} \succeq \mathbf{0}\left(\mathbf{W}_{k} \in \mathbf{H}_{+}^{N_{B}}\right)$ is the transmit covariance; $\mathbf{z}(t) \sim$ $C N(\mathbf{0}, \mathbf{V})$ is the generated $\mathrm{AN}, \mathbf{V} \succeq \mathbf{0}\left(\mathbf{V} \in \mathbf{H}_{+}^{N_{B}}\right)$ is the AN covariance. Under the assumption that all the communication links are undergoing quasi-static frequency-flat fading. The received signals at the BS, the $k$ th DL user, and the $m$ th eavesdropper are modeled as

$$
\begin{aligned}
& \mathbf{y}^{\mathrm{UL}}(t)=\mathbf{G}^{H} \mathbf{u}(t)+\underbrace{\mathbf{H}_{S I} \sum_{k=1}^{K} \mathbf{s}_{k}(t)}_{\text {self-interference }}+\underbrace{\mathbf{H}_{S I} \mathbf{z}(t)}_{\begin{array}{c}
\text { artificial noise } \\
\text { interference }
\end{array}}+\mathbf{n}^{\mathrm{UL}}, \\
& \mathbf{y}_{k}^{\mathrm{DL}}(t)=\mathbf{H}_{k}^{H} \mathbf{s}_{k}(t)+\underbrace{\sum_{i \neq k}^{K} \mathbf{H}_{k}^{H} \mathbf{s}_{i}(t)}_{\text {multiuser interference }}+\underbrace{\mathbf{H}_{k}^{H} \mathbf{z}(t)}_{\begin{array}{c}
\text { artificial noise } \\
\text { interference }
\end{array}}+\underbrace{\mathbf{F}_{k}^{H} \mathbf{u}(t)}_{\begin{array}{c}
\text { co-channel } \\
\text { interference }
\end{array}}+\mathbf{n}_{k}^{\mathrm{DL}},
\end{aligned}
$$

$$
k \in K,
$$

$\mathbf{y}_{m}^{\mathrm{E}}(t)=\sum_{k=1}^{K} \mathbf{L}_{m}^{H} \mathbf{s}_{k}(t)+\mathbf{E}_{m}^{H} \mathbf{u}(t)+\mathbf{L}_{m}^{H} \mathbf{z}(t)+\mathbf{n}_{m}^{\mathrm{E}}, \quad m \in M$,

respectively, where $M \in\{1, \cdots, M\} ; \mathbf{G} \in C^{N_{U} \times N_{B}}$, $\mathbf{H}_{k} \in C^{N_{B} \times N_{D}}$, and $\mathbf{L}_{m} \in C^{N_{B} \times N_{E}}$ are the channel matrices between the FD BS and the UL user, the DL user $k$, and the eavesdropper $m$, respectively; $\mathbf{F}_{k} \in C^{N_{U} \times N_{D}}$ and $\mathbf{E}_{m} \in C^{N_{U} \times N_{E}}$ are the channel matrices between the UL user and DL user $k$, and the eavesdropper $m$, resp.; $\mathbf{H}_{S I} \in \mathbb{C}^{N_{B} \times N_{B}}$ is the self-interference (SI) channel of the FD BS; $\mathbf{n}^{\mathrm{UL}} \sim C N\left(\mathbf{0}, \mathbf{I}_{N_{B}}\right), \mathbf{n}_{k}^{\mathrm{DL}} \sim C N\left(\mathbf{0}, \mathbf{I}_{N_{D}}\right)$, and $\mathbf{n}_{m}^{E} \sim C N\left(\mathbf{0}, \mathbf{I}_{N_{E}}\right)$ are standard additive white complex Gaussian noises at BS, $k$ th DL user, and $m$ th eavesdropper, resp.

\section{Problem formulation}

Our purpose is to design the (UL and DL) transmit and AN covariances $\boldsymbol{\Sigma},\left\{\mathbf{W}_{k}\right\}_{k=1}^{K}$ and $\mathbf{V}$ such that the minimum tolerable secure communication requirements for UL and DL channels can be ensured with the transmit power consumption as small as possible. Assuming perfect CSI at the FD BS, given $\boldsymbol{\Sigma},\left\{\mathbf{W}_{k}\right\}_{k=1}^{K}$ and $\mathbf{V}$, the channel capacity (bit/s/Hz) between the UL user and the FD BS is given by
$C^{\mathrm{UL}}=\log \left|\mathbf{I}+\left\{\rho \operatorname{Diag}\left[\mathbf{H}_{S I}\left(\sum_{k=1}^{K} \mathbf{W}_{k}+\mathbf{V}\right) \mathbf{H}_{S I}^{H}\right]+\mathbf{I}\right\}^{-1} \mathbf{G}^{H} \boldsymbol{\Sigma} \mathbf{G}\right|$,

where $\rho \operatorname{Diag}\left[\mathbf{H}_{S I}\left(\sum_{k=1}^{K} \mathbf{W}_{k}+\mathbf{V}\right) \mathbf{H}_{S I}^{H}\right]$ is the covariance of the SI elimination induced noise ([13], Eq. (4)), the main purpose of such a modelization is to isolate the resource allocation algorithm design from the specific implementation of self-interference mitigation; $0<\rho \leq 1$, a constant, denotes the noisiness of the SI elimination at the FD BS. The eavesdroppers overheard the uplink communication channel, and the mutual information between UL user and the $m$ th eavesdropper is given by

$C_{m}^{\mathrm{UL}-\mathrm{E}}=\log \left|\mathbf{I}+\left(\sum_{k=1}^{K} \mathbf{L}_{m}^{H} \mathbf{W}_{k} \mathbf{L}_{m}+\mathbf{L}_{m}^{H} \mathbf{V} \mathbf{L}_{m}+\mathbf{I}\right)^{-1} \mathbf{E}_{m}^{H} \boldsymbol{\Sigma} \mathbf{E}_{m}\right|, m \in M$.

Given $C^{\mathrm{UL}}$ and $C_{m}^{\mathrm{UL}-\mathrm{E}}$, the achievable secrecy rate of the UL channel can be given by [14]

$$
R^{\mathrm{UL}-\mathrm{S}}=\left[C^{\mathrm{UL}}-\max _{m \in M} C_{m}^{\mathrm{UL}-\mathrm{E}}\right]^{+}
$$

For the DL transmission, similar to the UL case, the achievable rate between FD BS and the $k$ th DL user can be given by

$C_{k}^{\mathrm{DL}}=\log \left|\mathbf{I}+\left(\sum_{i \neq k}^{K} \mathbf{H}_{k}^{H} \mathbf{W}_{i} \mathbf{H}_{k}+\mathbf{H}_{k}^{H} \mathbf{V} \mathbf{H}_{k}+\mathbf{F}_{k}^{H} \boldsymbol{\Sigma} \mathbf{F}_{k}+\mathbf{I}\right)^{-1} \mathbf{H}_{k}^{H} \mathbf{W}_{k} \mathbf{H}_{k}\right|, k \in K$.

Considering the existence of the interception among the DL channels, the mutual information between FD BS and the $m$ th eavesdropper for eavesdropping the desired DL user $k$ is given by

$C_{m, k}^{\mathrm{DL}-\mathrm{E}}=\log \left|\mathbf{I}+\left(\sum_{i \neq k}^{K} \mathbf{L}_{m}^{H} \mathbf{W}_{i} \mathbf{L}_{m}+\mathbf{L}_{m}^{H} \mathbf{V} \mathbf{L}_{m}+\mathbf{E}_{m}^{H} \mathbf{\Sigma} \mathbf{E}_{m}+\mathbf{I}\right)^{-1} \mathbf{L}_{m}^{H} \mathbf{W}_{k} \mathbf{L}_{m}\right|$,

$m \in M, k \in K$.

Given $C_{k}^{\mathrm{DL}}$ and $C_{m, k}^{\mathrm{DL}-\mathrm{E}}$, the achievable secrecy rate of the DL channel can be given by [15] 


$$
R_{k}^{\mathrm{DL}-\mathrm{S}}=\left[C_{k}^{\mathrm{DL}}-\max _{m \in M} C_{m, k}^{\mathrm{DL}-\mathrm{E}}\right]^{+}, k \in K
$$

In regard to the CSI of our focused FD MIMO communication system, the slowly time-varying channels are considered. For the UL channel, by performing handshaking between FD BS and UL user, the UL CSI can be reliably estimated at the FD BS through measuring the pilots from UL user in the handshaking signals via channel reciprocity at the beginning of each scheduling slot. Then, during the transmission, the UL user inserts the pilot signals termly into the data packets such that the FD BS is able to refine the CSI estimate of the UL user frequently. For the DL channels, similar to the UL channel, the handshaking is also executed between FD BS and the $k$ th DL user to promote the DL channel estimation at the beginning of each scheduling slot. Then, by sending the acknowledgement packets periodically to inform the FD BS of successful reception of data packets during the transmission, the CSI of the FD BS to the $k$ th DL user link can be perfectly known (with negligible estimation error) during whole transmission period. Consequently, here, we assume perfect CSI of both the $\mathrm{UL}$ and DL channels, $\mathbf{G}$ and $\left\{\mathbf{H}_{k}\right\}_{k=1}^{K}$. However, for the wiretap and co-channel interference channels, since there is no interaction between two different communication nodes during the transmission, their CSI at the FD BS is imperfect. To capture the effect of the imperfect CSI, a deterministic model $[16,17]$ is introduced to model the resulting CSI uncertainty, while the Gaussian estimation error model [18] will be left as future work. In particular, the CSI between the FD BS and the $m$ th eavesdropper, i.e., $\mathbf{L}_{m}, m \in M$, the CSI between the UL user and the $m$ th eavesdropper, i.e., $\mathbf{E}_{m}, m \in M$, the CSI between the UL user and the $k$ th DL user, i.e., $\mathbf{F}_{k}, k \in K$, are given as

$$
\begin{aligned}
& \mathbf{L}_{m}=\overline{\mathbf{L}}_{m}+\Delta \mathbf{L}_{m},\left\|\Delta \mathbf{L}_{m}\right\|_{F} \leq \varepsilon_{L, m}, m \in M, \\
& \mathbf{E}_{m}=\overline{\mathbf{E}}_{m}+\Delta \mathbf{E}_{m},\left\|\Delta \mathbf{E}_{m}\right\|_{F} \leq \varepsilon_{E, m}, m \in M, \\
& \mathbf{F}_{k}=\overline{\mathbf{F}}_{k}+\Delta \mathbf{F}_{k},\left\|\Delta \mathbf{F}_{k}\right\|_{F} \leq \varepsilon_{F, k}, k \in K,
\end{aligned}
$$

resp., where $\mathbf{L}_{m}, \mathbf{E}_{m}$, and $\mathbf{F}_{k}$ are the actual channel states at the FD BS and UL user, resp., while $\overline{\mathbf{L}}_{m}, \overline{\mathbf{E}}_{m}$, and $\overline{\mathbf{F}}_{k}$ are the channel estimation values, and $\Delta \mathbf{L}_{m}, \Delta \mathbf{E}_{m}$, and $\Delta \mathbf{F}_{m}$ are the channel estimation errors which are bounded in the deterministic regions $\left\|\Delta \mathbf{L}_{m}\right\|_{F},\left\|\Delta \mathbf{E}_{m}\right\|_{F}$, and $\left\|\Delta \mathbf{F}_{m}\right\|_{F}$, resp., for $\varepsilon_{L, m}>0, \varepsilon_{E, m}>0$, and $\varepsilon_{F, k}>0$.

Two optimization problems, UL and DL transmit power minimization, are considered in our resource allocation algorithm designs. Based on the analysis of the secrecy rates and CSI in above paragraphs, the first considered optimization problem minimizing the UL transmit power under the constraints of system secrecy rates is formulated as

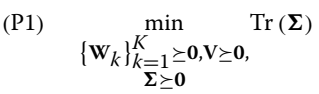

$$
\begin{aligned}
& \text { s.t. } \quad C 1: \underbrace{\log \left|\mathbf{I}+\mathbf{Q}^{-1} \mathbf{G}^{H} \boldsymbol{\Sigma} \mathbf{G}\right|-\max _{\substack{\mathbf{L}_{m} \in \mathbf{B}_{L, m} \\
\mathbf{E}_{m} \in \mathbf{B}_{E, m}}} \log \left|\mathbf{I}+\boldsymbol{\Phi}_{m}^{-1} \mathbf{E}_{m}^{H} \boldsymbol{\Sigma} \mathbf{E}_{m}\right|}_{R^{\mathrm{UL}}-\mathrm{S}} \geq \bar{R}^{\mathrm{UL}-\mathrm{S}, \forall m,} \\
& C 2: \underbrace{\max _{\mathbf{F}_{k} \in \mathbf{B}_{F, k}} \log \left|\mathbf{I}+\boldsymbol{\Omega}_{k}^{-1} \mathbf{H}_{k}^{H} \mathbf{W}_{k} \mathbf{H}_{k}\right|-\max _{\substack{\mathbf{E}_{m} \in \mathbf{B}_{E, m} \\
\mathbf{L}_{m} \in \mathbf{B}_{L, m}}} \log \left|\mathbf{I}+\Psi_{m, k}^{-1} \mathbf{L}_{m}^{H} \mathbf{W}_{k} \mathbf{L}_{m}\right|}_{R_{k}^{\text {DL-S }}} \\
& \geq \bar{R}_{k}^{\mathrm{DL}-\mathrm{S}}, \forall m, k, \\
& C 3: \operatorname{Tr}(\boldsymbol{\Sigma}) \leq P_{\max }^{\mathrm{UL}}, \quad C 4: \sum_{k=1}^{K} \operatorname{Tr}\left(\mathbf{W}_{k}\right)+\operatorname{Tr}(\mathbf{V}) \leq P_{\max }^{\mathrm{DL}}, \\
& C 5: \mathbf{W}_{k} \succeq \mathbf{0}, \mathbf{V} \succeq \mathbf{0}, \mathbf{\Sigma} \succeq \mathbf{0}, \forall k,
\end{aligned}
$$

where

$$
\begin{aligned}
& \mathbf{Q}=\rho \operatorname{Diag}\left[\mathbf{H}_{S I}\left(\sum_{k=1}^{K} \mathbf{W}_{k}+\mathbf{V}\right) \mathbf{H}_{S I}^{H}\right]+\mathbf{I}, \\
& \mathbf{\Phi}_{m}=\mathbf{L}_{m}^{H}\left(\sum_{k=1}^{K} \mathbf{W}_{k}+\mathbf{V}\right) \mathbf{L}_{m}+\mathbf{I}, \\
& \mathbf{\Omega}_{k}=\mathbf{H}_{k}^{H}\left(\sum_{i \neq k}^{K} \mathbf{W}_{i}+\mathbf{V}\right) \mathbf{H}_{k}+\mathbf{F}_{k}^{H} \boldsymbol{\Sigma} \mathbf{F}_{k}+\mathbf{I} \text { and } \\
& \mathbf{\Psi}_{m, k}=\mathbf{L}_{m}^{H}\left(\sum_{i \neq k}^{K} \mathbf{W}_{i}+\mathbf{V}\right) \mathbf{L}_{m}+\mathbf{E}_{m}^{H} \boldsymbol{\Sigma} \mathbf{E}_{m}+\mathbf{I} ; \\
& \mathbf{B}_{L, m}=\left\{\mathbf{L}_{m} \mid \mathbf{L}_{m}=\overline{\mathbf{L}}_{m}+\Delta \mathbf{L}_{m},\left\|\Delta \mathbf{L}_{m}\right\|_{F} \leq \varepsilon_{L, m}\right\}, \forall m, \\
& \mathbf{B}_{E, m}=\left\{\mathbf{E}_{m} \mid \mathbf{E}_{m}=\overline{\mathbf{E}}_{m}+\Delta \mathbf{E}_{m},\left\|\Delta \mathbf{E}_{m}\right\|_{F} \leq \varepsilon_{E, m}\right\}, \forall m \text { and } \\
& \mathbf{B}_{F, k}=\left\{\mathbf{F}_{k} \mid \mathbf{F}_{k}=\overline{\mathbf{F}}_{k}+\Delta \mathbf{F}_{k},\left\|\Delta \mathbf{F}_{k}\right\|_{F} \leq \varepsilon_{F, k}\right\}, \forall k ;
\end{aligned}
$$

$\bar{R}^{\mathrm{UL}-\mathrm{S}}>0$ and $\bar{R}_{k}^{\mathrm{DL}-\mathrm{S}}>0$ in $\mathrm{C} 1$ and C2, resp., specify the minimum required secrecy rate of the UL and $k$ th DL channel, resp.; $P_{\max }^{\mathrm{UL}}>0$ in $\mathrm{C} 3$ gives the maximum allowable UL transmit power of the UL user while $P_{\max }^{\mathrm{DL}}>0$ in $\mathrm{C} 4$ gives the maximum allowable DL transmit power of the FD BS; C5 implies the positive semi-definiteness of the optimization variables.

On the other hand, for the efficient resource allocation from the perspective of whole system, the DL transmit power minimization under same constraints as in problem (P1) can be given as

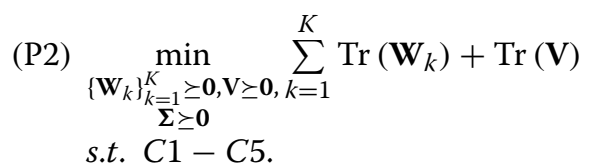

It is obvious that the aforementioned system design objectives in problems (P1) and (P2) are desirable for our system operator to provide the reliable quality of service 
(QoS) regarding secrecy communications for different users with the least possible power consumption. However, as mentioned above, each objective concerns only one aspect of the system and the two designed objectives even conflict with each other. For instance, large transmit power of the UL user provides high communication quality of the UL channel, and ensures reliable communication security to some extent. But, this will lead to severe co-channel interference to the DL users, degrade the communication performances of the DL channels. To circumvent it, the FD BS increases the DL transmit power to offset the adverse impact caused by co-channel interference and enhance the DL signal reception. Yet, higher FD BS transmission power results in strong SI which in turn impairing the reception of the signals intended for the FD BS. Hence, a non-trivial trade-off design for balancing two conflicting objectives arises in our considered multi-user FD MIMO communication system.

In this paper, we resort to the MOO mathematical framework to study the trade-off between the UL and DL transmission power via the concept of Pareto optimality [11], which can be given as

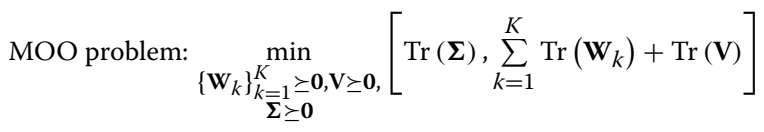

$$
\begin{aligned}
& \text { s.t. } C 1-C 5 \text {. }
\end{aligned}
$$

In order to facilitate solving, the weighted Tchebycheff method [19] is employed to convert the problem (14) into a weighted form MOO problem. We define such a problem as problem (P3), which is given by

$$
\begin{aligned}
& \text { (P3) } \min _{\substack{\left.\mathbf{W}_{k}\right\}^{K} \in=\mathbf{0}, \mathbf{V} \succeq \mathbf{0}, j=1,2 \\
\boldsymbol{\Sigma} \succeq \geq \mathbf{0}}}\left\{\lambda_{j}\left[\mathrm{O}_{j}(\mathbf{W}, \mathbf{V}, \mathbf{\Sigma})-\mathrm{O}_{j}^{*}\right]\right\} \\
& \text { s.t. } \mathrm{C} 1-C 5,
\end{aligned}
$$

where $\mathrm{O}_{1}(\mathbf{W}, \mathbf{V}, \boldsymbol{\Sigma})=\operatorname{Tr}(\boldsymbol{\Sigma}), \mathrm{O}_{2}(\mathbf{W}, \mathbf{V}, \boldsymbol{\Sigma})=$ $\sum_{k=1}^{K} \operatorname{Tr}\left(\mathbf{W}_{k}\right)+\operatorname{Tr}(\mathbf{V}), \mathrm{O}_{j}^{*}$ represents the optimal objective value with respect to (w.r.t) problems (P1) and (P2), $0<\lambda_{j}<1$, a constant with $\sum_{j=1}^{2} \lambda_{j}=1$, is the weight imposed on the $j$ th objective. Different pre-specified weights $\left\{\lambda_{j}\right\}_{j=1}^{2}$ are assigned to each of the objectives to show the preferences of the system operator. The optimal solutions of the MOO problem (P3) (by varying the weights) consist of the Pareto frontier or the Pareto optimal set, each point in the frontier denotes one preferential design of the system operator.

In fact, the MOO problem (P3) is a generalization of the single-objective optimization problems (P1) and (P2). In other words, problem (P3) is equivalent to problem (Pj) when $\lambda_{j}=1$ and $\lambda_{i}=0, i \neq j$. As a consequence, in the following section, we mainly focus on the methodology for solving the MOO problem (P3).

\section{Robust resource allocation algorithm design to MOOP}

It is obvious that problem (P3) is non-convex owe to the non-convex objective and constraints $\mathrm{C} 1$ and $\mathrm{C} 2$. Through the introduction of a new variable $\tau$, the objective can be transformed into a convex one, and the transformed problem of (P3) is given by

$$
\begin{array}{ll}
(\widehat{\mathrm{P}} 3) & \min _{\substack{\left\{\mathbf{W}_{k}\right\}_{k=1}^{K} \geq \mathbf{0}, \mathbf{V} \geq \mathbf{0}, \mathbf{\Sigma} \geq \mathbf{0}, \tau \geq 0}} \tau \\
& \text { s.t. } \mathrm{C} 1-C 5, \\
& \mathrm{C} 6: \lambda_{j}\left[\mathrm{O}_{j}(\mathbf{W}, \mathbf{V}, \boldsymbol{\Sigma})-\mathrm{O}_{j}^{*}\right] \leq \tau, \forall j .
\end{array}
$$

Next, we focus on dealing with the non-convex constraints $\mathrm{C} 1$ and $\mathrm{C} 2$. It is noteworthy that the terms on the left side of the inequalities of both constraints $\mathrm{C} 1$ and $\mathrm{C} 2$ are constructed in the form of the difference between two convex expressions. Moreover, the uncertainties of CSI also lead to the semiinfiniteness of the constraints $\mathrm{C} 1$ and $\mathrm{C} 2$, which further enhances the complexity of the non-convex constraints. Hence, reformulation is needed to transform them into the convex constraints with finite numbers. To this end, we first endeavor to dispose of constraint $\mathrm{C} 1$ while the processing of $\mathrm{C} 2$ can be performed in a similar way.

As regards constraint $\mathrm{C} 1$, introducing two additional auxiliary variables $\alpha_{1}$ and $\alpha_{2}$, it can be recast as

$$
C 1:\left\{\begin{array}{l}
C 1.1: \ln \left|\mathbf{I}+\mathbf{Q}^{-1} \mathbf{G}^{H} \mathbf{\Sigma} \mathbf{G}\right| \geq \alpha_{1}, \\
C 1.2: \max _{\substack{\mathbf{L}_{m} \in B_{L, m} \\
\mathbf{E}_{m} \in B_{E, m}}} \ln \left|\mathbf{I}+\mathbf{\Phi}^{-1} \mathbf{E}_{m}^{H} \boldsymbol{\Sigma} \mathbf{E}_{m}\right| \leq \alpha_{2}, \forall m, \\
C 1.3: \alpha_{1}-\alpha_{2} \geq \bar{R}^{\mathrm{UL}-\mathrm{S}} \ln 2 .
\end{array}\right.
$$

From (17), we note that the non-convexity caused by the difference of two logarithmic functions is settled. However, the newly formed constraints $\mathrm{C} 1.1$ and $\mathrm{C} 1.2$ are still appeared in non-convex forms. By adopting the first-order Taylor approximation, constraints $\mathrm{C} 1.1$ and $\mathrm{C} 1.2$ can be rewritten as

$$
\begin{aligned}
& \ln \left|\mathbf{I}+\mathbf{Q}^{-1} \mathbf{G}^{H} \mathbf{\Sigma} \mathbf{G}\right| \\
& =\ln |\boldsymbol{\Theta}|-\ln |\mathbf{Q}| \\
& \simeq \ln |\mathbf{\Theta}|-\ln \left|\mathbf{Q}_{0}\right|- \\
& \operatorname{Tr}\left\{\mathbf{Q}_{0}^{-1} \rho \operatorname{Diag}\left[\mathbf{H}_{S I}\left(\sum_{k=1}^{K} \mathbf{W}_{k}+\mathbf{V}-\sum_{k=1}^{K} \mathbf{W}_{k 0}-\mathbf{V}_{0}\right) \mathbf{H}_{S I}^{H}\right]\right\} \\
& \geq \alpha_{1},
\end{aligned}
$$




$$
\begin{aligned}
& \max _{\substack{\mathbf{L}_{m} \in B_{L, m} \\
\mathbf{E}_{m} \in B_{E, m}}} \ln \left|\mathbf{I}+\boldsymbol{\Phi}^{-1} \mathbf{E}_{m}^{H} \boldsymbol{\Sigma} \mathbf{E}_{m}\right| \\
= & \max _{\substack{\mathbf{L}_{m} \in B_{L, m} \\
\mathbf{E}_{m} \in B_{E, m}}}\left\{\ln \left|\boldsymbol{\Delta}_{m}\right|-\ln \left|\boldsymbol{\Phi}_{m}\right|\right\} \\
\simeq & \max _{\substack{\mathbf{L}_{m} \in B_{L, m} \\
\mathbf{E}_{m} \in B_{E, m}}}\left\{+\operatorname{Tr}\left[\boldsymbol{\Delta}_{m 0}^{-1}\left(\begin{array}{c}
\left.\mathbf{L}_{m}^{H}\left(\sum_{k=1}^{K} \mathbf{W}_{k}+\mathbf{V}-\sum_{k=1}^{K} \mathbf{W}_{k 0}-\mathbf{V}_{0}\right) \mathbf{L}_{m}\right) \\
\left.+\mathbf{E}_{m}^{H}\left(\boldsymbol{\Sigma}-\mathbf{\Sigma}_{0}\right) \mathbf{E}_{m}\right]-\ln \left|\boldsymbol{\Phi}_{m}\right|
\end{array}\right\}\right.\right. \\
\leq & \alpha_{2},
\end{aligned}
$$

respectively, where $\mathbf{W}_{k 0}, \mathbf{V}_{0}$, and $\boldsymbol{\Sigma}_{0}$ are the initial feasible points of the first-order Taylor approximation of the original problem (P3). For easy identification, we rename the transformed constraints in (18) and (19) as $\overline{C 1.1}$ and $\overline{C 1.2}$, respectively. The optimal solution can be obtained through iteration that updating $\mathbf{W}_{k 0}, \mathbf{V}_{0}$, and $\boldsymbol{\Sigma}_{0}$ from the optimal $\mathbf{W}_{k 0}^{*}, \mathbf{V}_{0}^{*}$, and $\boldsymbol{\Sigma}_{0}^{*}$ of the previous iteration by solving problem $(\widetilde{\mathrm{P} 3})$ as listed in (44). The iteration will be ended once $\mathbf{W}_{k 0}=\mathbf{W}_{k 0}^{*}, \mathbf{V}_{0}=\mathbf{V}_{0}^{*}$, and $\boldsymbol{\Sigma}_{0}=\boldsymbol{\Sigma}_{0}^{*}$ hold [20]; and

$$
\begin{aligned}
\boldsymbol{\Theta}= & \mathbf{Q}+\mathbf{G}^{H} \boldsymbol{\Sigma} \mathbf{G}=\rho \operatorname{Diag}\left[\mathbf{H}_{S I}\left(\sum_{k=1}^{K} \mathbf{W}_{k}+\mathbf{V}\right) \mathbf{H}_{S I}^{H}\right] \\
& +\mathbf{G}^{H} \mathbf{\Sigma} \mathbf{G}+\mathbf{I}, \\
\mathbf{Q}_{0}= & \rho \operatorname{Diag}\left[\mathbf{H}_{S I}\left(\sum_{k=1}^{K} \mathbf{W}_{k 0}+\mathbf{V}_{0}\right) \mathbf{H}_{S I}^{H}\right]+\mathbf{I}, \\
\boldsymbol{\Delta}_{m}= & \boldsymbol{\Phi}_{m}+\mathbf{E}_{m}^{H} \boldsymbol{\Sigma} \mathbf{E}_{m}=\mathbf{L}_{m}^{H}\left(\sum_{k=1}^{K} \mathbf{W}_{k}+\mathbf{V}\right) \mathbf{L}_{m} \\
& +\mathbf{E}_{m}^{H} \boldsymbol{\Sigma} \mathbf{E}_{m}+\mathbf{I}_{\text {and }} \\
\boldsymbol{\Delta}_{m 0}= & \mathbf{L}_{m}^{H}\left(\sum_{k=1}^{K} \mathbf{W}_{k 0}+\mathbf{V}_{0}\right) \mathbf{L}_{m}+\mathbf{E}_{m}^{H} \mathbf{\Sigma}_{0} \mathbf{E}_{m}+\mathbf{I} .
\end{aligned}
$$

Remark 2 Given a convex function $f(\mathbf{X})$, where $\mathbf{X}$ includes all the matrix variables associated with it. The implication $\tilde{f}(\mathbf{X}) \geq c \Rightarrow f(\mathbf{X}) \geq c$ holds, where $\tilde{f}(\mathbf{X})$ denotes the first-order Taylor approximation of $f(\mathbf{X})$ at any initial feasible point $\mathbf{X}_{0}$ and $c>0$ denotes any real constant, since $\tilde{f}(\mathbf{X}) \leq f(\mathbf{X})$ satisfies at any $\mathbf{X} \succeq \mathbf{0}$ and the equation holds if and only if $\mathbf{X}=\mathbf{X}_{0}$ [20]. Iff $(\mathbf{X})$ is a concave function, then the implication will be $\tilde{f}(\mathbf{X}) \leq c \Rightarrow$ $f(\mathbf{X}) \leq c$. In other words, for the convex function $-\ln |\mathbf{Q}|$ and the concave function $\ln \left|\Delta_{m}\right|$ in (18) and (19), respective, after performing the first-order Taylor approximation on them, we can conclude that if constraints $\overline{C 1.1}$ and $\overline{C 1.2}$ satisfied, then the constraints C1.1 and C1.2 in (17) must be satisfied too.
Now, we find that the constraint $\overline{C 1.1}$ is convex w.r.t. the optimization variables $\left\{\mathbf{W}_{k}\right\}_{k=1}^{K}$ and V, while constraint $\overline{C 1.2}$ still remains non-convex due to the channel uncertainty sets. Focusing on constraint $\overline{C 1.2}$, introducing additional slack variables $\alpha_{3}, \alpha_{4}, \alpha_{5}$, and $\alpha_{6}$, we have

$\overline{C 1.2}:\left\{\begin{array}{l}C 1.2 .1: \max _{\substack{\mathbf{L}_{m} \in B_{L, m} \\ \mathbf{E}_{m} \in B_{E, m}}} \ln \left|\mathbf{\Delta}_{m 0}\right| \leq \ln \alpha_{3}, \\ C 1.2 .2: \max _{\mathbf{L}_{m} \in B_{L, m}} \operatorname{Tr}\left[\boldsymbol{\Delta}_{m 0}^{-1} \mathbf{L}_{m}^{H}\left(\sum_{k=1}^{K} \mathbf{W}_{k}+\mathbf{V}-\sum_{k=1}^{K} \mathbf{W}_{k 0}-\mathbf{V}_{0}\right) \mathbf{L}_{m}\right] \leq \alpha_{4}, \\ C 1.2 .3: \max _{\mathbf{E}_{m} \in B_{E, m}} \operatorname{Tr}\left[\boldsymbol{\Delta}_{m 0}^{-1} \mathbf{E}_{m}^{H}\left(\boldsymbol{\Sigma}-\mathbf{\Sigma}_{0}\right) \mathbf{E}_{m}\right] \leq \alpha_{5}, \\ C 1.2 .4: \max _{\mathbf{L}_{m} \in B_{L, m}} \ln \left|\boldsymbol{\Phi}_{m}\right| \geq \ln \alpha_{6}, \\ C 1.2 .5: \ln \alpha_{3}+\alpha_{4}+\alpha_{5}-\ln \alpha_{6} \leq \alpha_{2} .\end{array}\right.$

Considering that most of the constraints in (20) still remain non-convex. Firstly, we make a reformulation about $\mathrm{C} 1.2 .1$ as in the following proposition.

Proposition 1 The inequality constraint C1.2.1 can be equivalently expressed as $\ln \left|\boldsymbol{\Delta}_{m 0}\right| \leq \ln \alpha_{3}, \forall \mathbf{L}_{m} \in$ $B_{L, m}, \mathbf{E}_{m} \in B_{E, m}$. Then, we have the following implication

$$
\begin{aligned}
& \ln \left|\mathbf{\Delta}_{m 0}\right| \leq \ln \alpha_{3}, \forall \mathbf{L}_{m}, \mathbf{E}_{m} \\
\Rightarrow & \operatorname{Tr}\left[\mathbf{L}_{m}^{H}\left(\sum_{k=1}^{K} \mathbf{W}_{k 0}+\mathbf{V}_{0}\right) \mathbf{L}_{m}\right] \\
& +\operatorname{Tr}\left(\mathbf{E}_{m}^{H} \mathbf{\Sigma}_{0} \mathbf{E}_{m}\right) \leq \alpha_{3}-1, \forall \mathbf{L}_{m}, \mathbf{E}_{m} .
\end{aligned}
$$

Proof The equivalence transformation of the constraint C1.2.1 is easy to understand. Here, we devote ourselves to the proof of the implication (21). Before the start of the proof, consider such a lemma which will be used to assist the convex transformation of constraint C1.2.1.

\section{Lemma 1 (Golub and Loan [21]) It holds true that}

$$
\operatorname{det}(\mathbf{I}+\mathbf{A}) \geq 1+\operatorname{Tr}(\boldsymbol{A})
$$

for any $\boldsymbol{A} \geq \mathbf{0}$, the equation holds if and only if $\operatorname{Rank}(\boldsymbol{A})=$ 1 holds.

Based on Lemma 1, we have

$$
\begin{aligned}
\left|\boldsymbol{\Delta}_{m 0}\right| & =\left|\mathbf{I}+\mathbf{L}_{m}^{H}\left(\sum_{k=1}^{K} \mathbf{W}_{k 0}+\mathbf{V}_{0}\right) \mathbf{L}_{m}+\mathbf{E}_{m}^{H} \mathbf{\Sigma}_{0} \mathbf{E}_{m}\right| \\
& \geq 1+\operatorname{Tr}\left[\mathbf{L}_{m}^{H}\left(\sum_{k=1}^{K} \mathbf{W}_{k 0}+\mathbf{V}_{0}\right) \mathbf{L}_{m}\right]+\operatorname{Tr}\left[\mathbf{E}_{m}^{H} \mathbf{\Sigma}_{0} \mathbf{E}_{m}\right] .
\end{aligned}
$$

The transformation in (22) is an approximation about $\left|\Delta_{m 0}\right|$ since the rank one condition stated in Lemma 1 can not be guaranteed here. The main purpose that 
we consider this lemma is to replace the determinant operations with the trace operations. Then, with the trace operations, we can overcome the semiinfiniteness caused by the channel uncertainties conveniently. Another effective method in dealing with the determinant operation has ever been proposed in [22], in which the Taylor series expansion was adopted and the global optimal solution was obtained at the convergence of the iterative algorithm. Replacing (22) into the constraint C1.2.1, we have the following relaxation for C1.2.1:

$$
\begin{aligned}
& \ln \left|\mathbf{\Delta}_{m 0}\right| \leq \ln \alpha_{3}, \forall \mathbf{L}_{m} \in B_{L, m}, \mathbf{E}_{m} \in B_{E, m} \\
\Leftrightarrow & \left|\boldsymbol{\Delta}_{m 0}\right| \leq \alpha_{3} \\
\Rightarrow & \operatorname{Tr}\left[\mathbf{L}_{m}^{H}\left(\sum_{k=1}^{K} \mathbf{W}_{k 0}+\mathbf{V}_{0}\right) \mathbf{L}_{m}\right]+\operatorname{Tr}\left(\mathbf{E}_{m}^{H} \mathbf{\Sigma}_{0} \mathbf{E}_{m}\right) \\
& \leq \alpha_{3}-1, \forall \mathbf{L}_{m}, \mathbf{E}_{m} .
\end{aligned}
$$

The proof of Proposition 1 is thus finished.

After this, we endeavor in overcoming the semiinfiniteness of constraint C1.2.1. To this end, the Sprocedure [23] is usually employed to convert it into a finite number of linear matrix inequality (LMI). Learning that $\operatorname{Tr}\left(\mathbf{A}^{H} \mathbf{B C D}\right)=\operatorname{vec}(\mathbf{A})^{H}\left(\mathbf{D}^{T} \otimes \mathbf{B}\right) \operatorname{vec}(\mathbf{C})$, based on Proposition 1, the constraint $\mathrm{C} 1.2 .1$ can be equivalently rewritten as

$$
\begin{aligned}
& \Delta \mathbf{l}_{m}^{H} \mathbf{S}_{0} \Delta \mathbf{l}_{m}+2 \operatorname{Re}\left\{\mathbf{l}_{m}^{H} \mathbf{S}_{0} \Delta \mathbf{l}_{m}\right\}+\overline{\mathbf{l}}_{m}^{H} \mathbf{S}_{0} \overline{\mathbf{l}}_{m}+\Delta \mathbf{e}_{m}^{H} \mathbf{T}_{0} \Delta \mathbf{e}_{m}+ \\
& 2 \operatorname{Re}\left\{\overline{\mathbf{e}}_{m}^{H} \mathbf{T}_{0} \Delta \mathbf{e}_{m}\right\}+\overline{\mathbf{e}}_{m}^{H} \mathbf{T}_{0} \overline{\mathbf{e}}_{m}-\alpha_{3}+1 \leq 0
\end{aligned}
$$

where $\overline{\mathbf{l}}_{m}=\operatorname{vec}\left(\overline{\mathbf{L}}_{m}\right), \Delta \mathbf{l}_{m}=\operatorname{vec}\left(\Delta \mathbf{L}_{m}\right), \overline{\mathbf{e}}_{m}=\operatorname{vec}\left(\overline{\mathbf{E}}_{m}\right)$, $\Delta \mathbf{e}_{m}=\operatorname{vec}\left(\Delta \mathbf{E}_{m}\right), \mathbf{l}_{m}=\overline{\mathbf{l}}_{m}+\Delta \mathbf{l}_{m}, \mathbf{e}_{m}=\overline{\mathbf{e}}_{m}+\Delta \mathbf{e}_{m}, \mathbf{S}_{0}=$ $\mathbf{I} \otimes\left(\sum_{k=1}^{K} \mathbf{W}_{k 0}+\mathbf{V}_{0}\right)$ and $\mathbf{T}_{0}=\mathbf{I} \otimes \mathbf{\Sigma}_{0}$. Since the uncertainties of $\mathbf{L}_{m}$ and $\mathbf{E}_{m}$ are taken into account separately, here, we first deal with $\mathbf{L}_{m}$ with the aid of S-procedure, and (24) is equivalently re-expressed as

$$
\begin{aligned}
{\left[\begin{array}{cc}
\delta_{L, m} \mathbf{I}-\mathbf{S}_{0} & -\mathbf{S}_{0}^{H} \overline{\mathbf{l}}_{m} \\
-\overline{\mathbf{l}}_{m}^{H} \mathbf{S}_{0} & -\delta_{L, m} \varepsilon_{L, m}^{2}-a_{m}
\end{array}\right] } & \geq \mathbf{0}, \exists \delta_{L, m} \\
& \geq 0, \forall \mathbf{e}_{m} \in \mathbf{B}_{e, m}, \forall m,
\end{aligned}
$$

where $a_{m}=\Delta \mathbf{e}_{m}^{H} \mathbf{T}_{0} \Delta \mathbf{e}_{m}+2 \operatorname{Re}\left\{\overline{\mathbf{e}}_{m}^{H} \mathbf{T}_{0} \Delta \mathbf{e}_{m}\right\}+$ $\overline{\mathbf{e}}_{m}^{H} \mathbf{T}_{0} \overline{\mathbf{e}}_{m}+\overline{\mathbf{l}}_{m}^{H} \mathbf{S}_{0} \overline{\mathbf{l}}_{m}-\alpha_{3}+1$ and $\mathbf{B}_{e, m}=$ $\left\{\mathbf{e}_{m} \mid \mathbf{e}_{m}=\overline{\mathbf{e}}_{m}+\Delta \mathbf{e}_{m},\left\|\mathbf{e}_{m}\right\|_{2} \leq \varepsilon_{E, m}\right\}$. Then, we resolve the semi-infiniteness caused by the imperfectness of channel $\mathbf{E}_{m}$ via utilizing the extended S-procedure [24] which is summarized in the following lemma.

\section{Lemma 2 The relation}

$$
\left[\begin{array}{cc}
\mathbf{A} & \mathbf{B}+\mathbf{C X} \\
(\mathbf{B}+\mathbf{C X})^{H} & \mathbf{D}+\mathbf{X}^{H} \mathbf{F}+\mathbf{F}^{H} \mathbf{X}+\mathbf{X}^{H} \mathbf{G X}
\end{array}\right] \geq \mathbf{0}
$$

Is valid, if and only if

$$
\left[\begin{array}{ccc}
\mathbf{A} & \mathbf{B} & \mathbf{C} \\
\mathbf{B}^{H} & \mathbf{D}-\delta \mathbf{I} & \mathbf{F}^{H} \\
\mathbf{C}^{H} & \mathbf{F} & \mathbf{G}+\delta \mathbf{E}
\end{array}\right] \geq \mathbf{0}, \exists \delta \geq 0 .
$$

According to Lemma 2, by introducing a new variable $\delta_{E, m} \geq 0$, (25) can be further reformulated as a single convex constraint given by

$$
\begin{aligned}
\widetilde{C 1.2 .1}: & {\left[\begin{array}{ccc}
\delta_{L, m} \mathbf{I}-\mathbf{S}_{0} & -\mathbf{S}_{0}^{H} \overline{\mathbf{l}}_{m} & \mathbf{0} \\
-\overline{\mathbf{l}}_{m}^{H} \mathbf{S}_{0} & b_{m}-\delta_{E, m} & \overline{\mathbf{e}}_{m}^{H} \mathbf{T}_{0} \\
\mathbf{0} & \mathbf{T}_{0}^{H} \overline{\mathbf{e}}_{m} & \mathbf{T}_{0}+\delta_{E, m}\left(\varepsilon_{E, m}^{-1}\right)^{2} \mathbf{I}
\end{array}\right] } \\
& \geq \mathbf{0}, \forall \delta_{L, m} \geq 0, \delta_{E, m} \geq 0,
\end{aligned}
$$

where. Similarly, make full use of the S-procedure, the constraints $\mathrm{C} 1.2 .2, \mathrm{C} 1.2 .3$, and $\mathrm{C} 1.2 .4$ can also be equivalently transformed as

$$
\begin{aligned}
& \widetilde{C 1.2 .2}: {\left[\begin{array}{cc}
\delta_{L, m}^{\prime} \mathbf{I}-\mathbf{S}_{1} & -\mathbf{S}_{1}^{H} \overline{\mathbf{l}}_{m} \\
-\mathbf{l}_{m}^{H} \mathbf{S}_{1} & -\delta_{L, m}^{\prime} \varepsilon_{L, m}^{2}-\overline{\mathbf{l}}_{m}^{H} \mathbf{S}_{1} \overline{\mathbf{l}}_{m}+\alpha_{4}
\end{array}\right] } \\
& \geq \mathbf{0}, \forall \delta_{L, m}^{\prime} \geq 0, m, \\
& \widetilde{C 1.2 .3}: {\left[\begin{array}{cc}
\delta_{E, m}^{\prime} \mathbf{I}-\mathbf{S}_{2} & -\mathbf{S}_{2}^{H} \overline{\mathbf{e}}_{m} \\
-\overline{\mathbf{e}}_{m}^{H} \mathbf{S}_{2} & -\delta_{E, m}^{\prime} \varepsilon_{E, m}^{2}-\overline{\mathbf{e}}_{m}^{H} \mathbf{S}_{2} \overline{\mathbf{e}}_{m}+\alpha_{5}
\end{array}\right] } \\
& \geq \mathbf{0}, \forall \delta_{E, m}^{\prime} \geq 0, m, \\
& \widetilde{C 1.2 .4}: {\left[\begin{array}{cc}
\delta_{L, m}^{\prime \prime} \mathbf{I}+\mathbf{S}_{3} & \mathbf{S}_{3}^{H} \overline{\mathbf{l}}_{m} \\
\overline{\mathbf{l}}_{m}^{H} \mathbf{S}_{3} & -\delta_{L, m}^{\prime \prime} \varepsilon_{L, m}^{2}+\overline{\mathbf{l}}_{m}^{H} \mathbf{S}_{3} \overline{\mathbf{l}}_{m}-\alpha_{6}+1
\end{array}\right] } \\
& \geq \mathbf{0}, \forall \delta_{L, m}^{\prime \prime} \geq 0, m,
\end{aligned}
$$

where $\mathbf{S}_{1}=\left(\boldsymbol{\Delta}_{m 0}^{-1}\right) \otimes\left(\sum_{k=1}^{K} \mathbf{W}_{k}+\mathbf{V}-\sum_{k=1}^{K} \mathbf{W}_{k 0}-\mathbf{V}_{0}\right)$, $\mathbf{S}_{2}=\left(\boldsymbol{\Delta}_{m 0}^{-1}\right)^{T} \otimes\left(\boldsymbol{\Sigma}-\boldsymbol{\Sigma}_{0}\right)$ and $\mathbf{S}_{3}=\mathbf{I} \otimes\left(\sum_{k=1}^{K} \mathbf{W}_{k}+\mathbf{V}\right)$. So far, the convex transformation of constraint $\mathrm{C} 1$ is finished and summarized as $\widetilde{C} 1$.

$$
\widetilde{C 1}:\left\{\begin{array}{l}
\overline{C 1.1}, \\
\widetilde{C 1.2 .1}, \widetilde{C 1.2 .2}, \widetilde{C 1.2 .3}, \widetilde{C 1.2 .4}, C 1.2 .5, \\
C 1.3 .
\end{array}\right.
$$

As seen from the expression of constraint C2, it appears to be more complicated than constraint C1. However, thanks to the techniques exploited in processing constraint $\mathrm{C} 1$, similar methods can be applied effectively in handling constraint $\mathrm{C} 2$. Firstly, introducing the auxiliary variables $\beta_{1}$ and $\beta_{2}$, we obtain 
$C 2: \begin{cases}C 2.1: \ln \left|\mathbf{I}+\mathbf{\Omega}_{k}^{-1} \mathbf{H}_{k}^{H} \mathbf{W}_{k} \mathbf{H}_{k}\right| \geq \beta_{1}, & \forall \mathbf{F}_{k} \in \mathbf{B}_{F, k}, k, \\ C 2.2: \ln \left|\mathbf{I}+\mathbf{\Psi}_{m, k}^{-1} \mathbf{L}_{m}^{H} \mathbf{W}_{k} \mathbf{L}_{m}\right| \leq \beta_{2}, & \forall \mathbf{L}_{m} \in \mathbf{B}_{L, k}, \mathbf{E}_{m} \in \mathbf{B}_{E, k}, m, k, \\ C 2.3: \beta_{1}-\beta_{2} \geq \bar{R}_{k}^{\mathrm{LL}-\mathrm{S}} \ln 2, \quad & \forall k,\end{cases}$

where $\boldsymbol{\Omega}_{k}$ and $\boldsymbol{\Psi}_{m, k}$ are what presented in above context. Next, for constraint C2.1 and C2.2, adopting one-order Taylor approximation, we have

$$
\begin{aligned}
C 2.1: \ln \mid & \mathbf{I}+\boldsymbol{\Omega}_{k}^{-1} \mathbf{H}_{k}^{H} \mathbf{W}_{k} \mathbf{H}_{k} \mid \\
= & \ln |\boldsymbol{\Pi}|-\ln \left|\boldsymbol{\Omega}_{k}\right| \\
\simeq & \ln |\boldsymbol{\Pi}|-\ln \left|\boldsymbol{\Omega}_{k 0}\right| \\
& -\operatorname{Tr}\left\{\boldsymbol { \Omega } _ { k 0 } ^ { - 1 } \left[\mathbf{H}_{k}^{H}\left(\sum_{i \neq k}^{K} \mathbf{W}_{k}+\mathbf{V}-\sum_{i \neq k}^{K} \mathbf{W}_{k 0}-\mathbf{V}_{0}\right) \mathbf{H}_{k}\right.\right. \\
& \left.\left.+\mathbf{F}_{k}^{H}\left(\mathbf{\Sigma}-\boldsymbol{\Sigma}_{0}\right) \mathbf{F}_{k}\right]\right\} \\
\geq & \beta_{1}, \forall \mathbf{F}_{k} \in \mathbf{B}_{F, k}, k \\
& \\
C 2.2: \ln & \left|\mathbf{I}+\mathbf{\Psi}_{m, k}^{-1} \mathbf{L}_{m}^{H} \mathbf{W}_{k} \mathbf{L}_{m}\right| \\
= & \ln \left|\mathbf{\Delta}_{m}\right|-\ln \left|\mathbf{\Psi}_{m, k}\right| \\
\simeq & \ln \left|\mathbf{\Delta}_{m 0}\right|+\operatorname{Tr}\left\{\mathbf { \Delta } _ { m 0 } ^ { - 1 } \left[\mathbf{L}_{m}^{H}\left(\sum_{k=1}^{K} \mathbf{W}_{k}+\mathbf{V}-\sum_{k=1}^{K} \mathbf{W}_{k 0}-\mathbf{V}_{0}\right) \mathbf{L}_{m}\right.\right. \\
& \left.\left.+\mathbf{E}_{m}^{H}\left(\mathbf{\Sigma}-\mathbf{\Sigma}_{0}\right) \mathbf{E}_{m}\right]\right\} \\
& -\ln \left|\mathbf{\Psi}_{m, k}\right| \\
\leq & \beta_{2}, \forall \mathbf{L}_{m} \in \mathbf{B}_{L, k}, \mathbf{E}_{m} \in \mathbf{B}_{E, k}, m, k
\end{aligned}
$$

where $\boldsymbol{\Pi}=\mathbf{H}_{k}^{H}\left(\sum_{k=1}^{K} \mathbf{W}_{k}+\mathbf{V}\right) \mathbf{H}_{k}+\mathbf{F}_{k}^{H} \boldsymbol{\Sigma} \boldsymbol{F}_{k}+\mathbf{I}, \boldsymbol{\Omega}_{k 0}=$ $\mathbf{H}_{k}^{H}\left(\sum_{i \neq k}^{K} \mathbf{W}_{k 0}+\mathbf{V}_{0}\right) \mathbf{H}_{k}+\mathbf{F}_{k}^{H} \boldsymbol{\Sigma}_{0} \mathbf{F}_{k}+\mathbf{I}$, and $\boldsymbol{\Delta}_{m}$ and $\boldsymbol{\Delta}_{m 0}$ are given above. Then, let us deal with the non-convexity caused by the channel uncertainties. By introducing auxiliary variables $\beta_{3}, \beta_{4}$, and $\beta_{5}$, the constraint C2.1 in (34) can be recast as

$$
\begin{aligned}
& C 2.1:\left\{\begin{array}{l}
C 2.1 .1: \ln |\boldsymbol{\Pi}| \geq \ln \beta_{3}, \\
C 2.1 .2: \ln \left|\boldsymbol{\Omega}_{k 0}\right| \leq \ln \beta_{4}, \\
C 2.1 .3: \operatorname{Tr}\left[\boldsymbol{\Omega}_{k 0}^{-1} \mathbf{F}_{k}^{H}\left(\boldsymbol{\Sigma}-\boldsymbol{\Sigma}_{0}\right) \mathbf{F}_{k}\right] \leq \beta_{5}, \\
C 2.1 .4: \ln \beta_{3}-\ln \beta_{4}-\beta_{5} \\
\quad-\operatorname{Tr}\left[\boldsymbol{\Omega}_{k 0}^{-1} \mathbf{H}_{k}^{H}\left(\sum_{i \neq k}^{K} \mathbf{W}_{k}+\mathbf{V}-\sum_{i \neq k}^{K} \mathbf{W}_{k 0}-\mathbf{V}_{0}\right) \mathbf{H}_{k}\right] \geq \beta_{1},
\end{array}\right. \\
& \forall \mathbf{F}_{k} \in \mathbf{B}_{F, k}, k \text {. }
\end{aligned}
$$

Performing the S-procedure w.r.t the uncertainty set $\mathbf{B}_{F, k}$, and $\mathrm{C} 2.1 .1, \mathrm{C} 2.1 .2$ and $\mathrm{C} 2.1 .3$ are equivalently transformed into

$$
\begin{aligned}
\widetilde{C 2.1 .1}: & \left.\begin{array}{cc}
\delta_{F, k} \mathbf{I}+\mathbf{T} & \mathbf{T}^{H} \overline{\mathbf{f}}_{k} \\
-{ }^{H} \mathbf{T} & -\delta_{F, k} \varepsilon_{F, k}^{2}+\overline{\mathbf{f}}_{k}^{H}-\overline{\mathbf{T}}_{k} \\
\mathbf{f}_{k} \mathbf{T} & +\operatorname{Tr}\left[\mathbf{H}_{k}^{H}\left(\sum_{k=1}^{K} \mathbf{w}_{k}+\mathbf{v}\right) \mathbf{H}_{k}\right]+1-\beta_{3}
\end{array}\right] \geq \mathbf{0}, \\
& \forall k, \exists \delta_{F, k} \geq 0,
\end{aligned}
$$

$$
\begin{aligned}
\widetilde{C 2.1 .2}\left[\begin{array}{cc}
\delta_{F, k}^{\prime} \mathbf{I}-\boldsymbol{\Sigma}_{0} & -\boldsymbol{\Sigma}_{0} \overline{\mathbf{f}}_{k} \\
-\overline{\mathbf{f}}_{k}^{H} \boldsymbol{\Sigma}_{0} & -\operatorname{Tr}\left[\mathbf{H}_{k}^{H}\left(\sum_{i \neq k}^{K} \mathbf{w}_{k 0}+\mathbf{v}_{0}\right) \mathbf{H}_{k}\right]-1+\beta_{4}-\overline{\mathbf{f}}_{k}^{H} \boldsymbol{\Sigma}_{0} \overline{\mathbf{f}}_{k}
\end{array}\right] \geq \mathbf{0}, \\
\quad \forall k, \exists \delta_{F, k}^{\prime} \geq 0,
\end{aligned}
$$

$$
\begin{aligned}
\widetilde{C 2.1 .3}: & {\left[\begin{array}{cc}
\delta_{F, k}^{\prime \prime} \mathbf{I}-\mathbf{U}_{k} & -\mathbf{U}_{k}^{H} \overline{\mathbf{f}}_{k} \\
-\overline{\mathbf{f}}_{k}^{H} \mathbf{U}_{k} & -\delta_{F, k}^{\prime \prime} \varepsilon_{F, k}^{2}-\overline{\mathbf{f}}_{k}^{H} \mathbf{U}_{k} \overline{\mathbf{f}}_{k}+\beta_{5}
\end{array}\right] \geq \mathbf{0}, } \\
& \forall k, \exists \delta_{F, k}^{\prime \prime} \geq 0,
\end{aligned}
$$

where $\mathbf{f}_{k}=\overline{\mathbf{f}}_{k}+\Delta \mathbf{f}_{k}=\operatorname{vec}\left(\mathbf{F}_{k}\right), \mathbf{T}=\mathbf{I} \otimes \mathbf{\Sigma}$ and $\mathbf{U}_{k}=\left(\boldsymbol{\Omega}_{k 0}^{-1}\right)^{T} \otimes\left(\boldsymbol{\Sigma}-\boldsymbol{\Sigma}_{0}\right)$. After this, the constraint C2.1 is converted to the convex form w.r.t its variables, which is depicted as follows:

$$
C 2.1:\left\{\begin{array}{l}
\widetilde{C 2.1 .1} \\
\widetilde{C 2.1 .2} \\
\overparen{C 2.1 .3} \\
C 2.1 .4
\end{array}\right.
$$

Similar to (36), constraint C2.2 can be recast as

$$
\begin{aligned}
C 2.2: & \left\{\begin{array}{l}
C 2.2 .1: \ln \left|\boldsymbol{\Psi}_{m, k}\right| \geq \ln \beta_{6}, \\
C .2 .2 .2: \ln \alpha_{3}+\alpha_{4}+\alpha_{5}-\ln \beta_{6} \leq \beta_{2},
\end{array}\right. \\
& \forall \mathbf{L}_{m} \in \mathbf{B}_{L, m}, \mathbf{E}_{m} \in \mathbf{B}_{E, m}, m, k,
\end{aligned}
$$

where $\beta_{6}$ is the newly introduced auxiliary variable. Then, according to S-procedure and its extension Lemma 2, C2.2.1 can be reduced to a single convex constraint given by

$$
\begin{aligned}
\widetilde{C 2.2 .1}: & {\left[\begin{array}{ccc}
\delta_{L, \underline{m}}^{\prime \prime} \mathbf{I}+\mathbf{S} & \mathbf{S}^{H} \overline{\mathbf{l}}_{m} & \mathbf{0} \\
\overline{\mathbf{l}}_{m}^{H} \mathbf{S} & c_{m}+1-\beta_{6} & \overline{\mathbf{e}}_{m}^{H} \mathbf{T} \\
\mathbf{0} & \mathbf{T}^{H} \overline{\mathbf{e}}_{m} & \mathbf{T}+\delta_{L, m}^{\prime \prime} \varepsilon_{E, m}^{-2} \mathbf{I}
\end{array}\right] \geq \mathbf{0}, } \\
& \exists \delta_{L, m}^{\prime \prime \prime} \geq 0, \delta_{E, m}^{\prime \prime} \geq 0, \forall m,
\end{aligned}
$$

where $\mathbf{S}=\mathbf{I} \otimes\left(\sum_{i \neq k}^{K} \mathbf{W}_{k}+\mathbf{V}\right)$ and $c_{m}=\overline{\mathbf{e}}_{m}^{H} \mathbf{T} \overline{\mathbf{e}}_{m}+\overline{\mathbf{l}}_{m}^{H} \mathbf{S} \overline{\mathbf{l}}_{m}-$ $\delta_{L, m}^{\prime \prime \prime} \varepsilon_{L, m}^{2}-\delta_{E, m}^{\prime \prime}$. Thus, for the original constraint C2, we 
have the following convex reformulation $\widetilde{C 2}$ composed of several sub-constraints

$$
\widetilde{C 2}:\left\{\begin{array}{l}
\widetilde{C 2.1 .1}, \widetilde{C 2.1 .2}, \widetilde{C 2.1 .3}, C 2.1 .4, \\
\overparen{C 2.2 .1}, C 2.2 .2, \\
C 2.3 .
\end{array}\right.
$$

Then, replacing constraints $\mathrm{C} 1$ and $\mathrm{C} 2$ by $\widetilde{C 1}$ and $\widetilde{C 2}$, respectively, problem $(\widehat{\mathrm{P} 3})$ can be transformed into the following convex problem with finite number of constraints:

$$
\begin{aligned}
(\widetilde{\mathrm{P}}): & \min _{\substack{\mathbf{W} \geq \mathbf{0}, \mathbf{V} \geq \mathbf{0}, \mathbf{\Sigma} \geq \mathbf{0}, \tau \geq 0, \alpha, \beta,\left\{\delta_{k}\right\}}} \tau \\
& \left\{\delta_{m=1}^{M}\right\}_{m=1}^{M}, \\
\text { s.t. } & \stackrel{\mathrm{C} 1, \bar{C} 2, C 3-C 6,}{\widetilde{C}},
\end{aligned}
$$

where $\alpha=\left\{\alpha_{1}, \alpha_{2}, \alpha_{3}, \alpha_{4}, \alpha_{5}, \alpha_{6}\right\}, \beta=\left\{\beta_{1}, \beta_{2}, \beta_{3}, \beta_{4}\right.$, $\left.\beta_{5}, \beta_{6}\right\}, \delta_{k}=\left\{\delta_{F, k}, \delta_{F, k}^{\prime}, \delta_{F, k}^{\prime \prime}\right\}$, and $\delta_{m}=\left\{\delta_{L, m}, \delta_{L, m}^{\prime}, \delta_{L, m}^{\prime \prime}\right.$, $\left.\delta_{L, m}^{\prime \prime \prime}, \delta_{E, m}, \delta_{E, m}^{\prime}, \delta_{E, m}^{\prime \prime}\right\}$. It is worthy to note that problem ( $\widetilde{\mathrm{P}} 3)$ can be solved efficiently by adopting the off-the-shelf convex optimization tools, such as SeDuMi (or CVX) [25]. Hence, the specific trade-off design scheme is obtained by choosing preferable weighted value $\lambda_{j}$. On the other hand, the approximate optimal solution for each single-objective optimization problem is also obtained by solving the special case of problem ( $\widetilde{\mathrm{P}} 3)$. Finally, an iterative algorithm is derived on the basis of the above procedures and the details are summarized in Algorithm 1.

Feasibility assurance about the initial point $\left\{\left\{\mathbf{W}_{k 0}\right\}_{k=1}^{K}, \mathbf{V}_{0}, \Sigma_{0}\right\}$ : The common way that preset $\left\{\left\{\mathbf{W}_{k 0}\right\}_{k=1}^{K}, \mathbf{V}_{0}, \Sigma_{0}\right\}$ is to initialize each one in it as zeros or the diagonal matrix with full transmission power uniformly distributed on each element. However, due to the fact that the initialization satisfy constraints $\mathrm{C} 3, \mathrm{C} 4$, and $\mathrm{C} 5$ in (12) may not be feasible to constraints $\mathrm{C} 1$ and $\mathrm{C} 2$, which will possibly result in a poor convergence performance of our proposed algorithm and better objective values compared to the optimal of the original problem. Therefore, to circumvent such a defect, the feasibility assurance procedure need to be injected in obtaining the feasible initial point. Particularly, we consider the following problem extracted from (P1) in (12)

$$
\begin{gathered}
\max _{\left\{\mathbf{W}_{k}\right\}_{k=1}^{K}, \mathbf{V}, \Sigma} \min _{k+1}\left\{R^{\mathrm{UL}-\mathrm{S}}-\bar{R}^{\mathrm{UL}-\mathrm{S}}, R_{k}^{\mathrm{DL}-\mathrm{S}}-\bar{R}_{k}^{\mathrm{DL}-\mathrm{S}}\right\} \\
\text { s.t. } \\
\text { C3, C4, C5. }
\end{gathered}
$$

Given the initial point, problem (45) can be solved effectively through the methods mentioned above. Then, the resulting optimal point $\left\{\left\{\mathbf{W}_{k 0}^{*}\right\}_{k=1}^{K}, \mathbf{V}_{0}^{*}, \Sigma_{0}^{*}\right\}$ which leads to non-negative objective value of problem (45) can be selected as the feasible initial point.

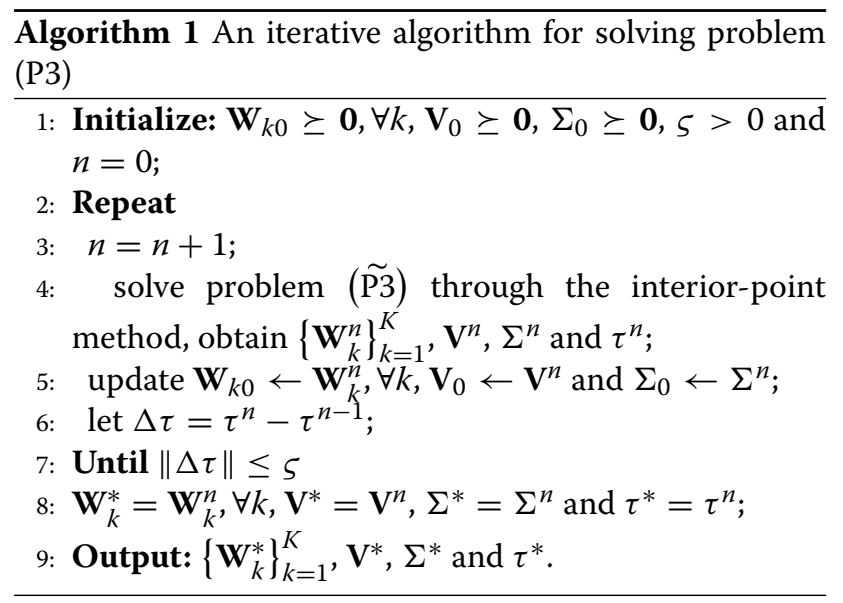

\section{Simulation results}

In this section, simulation results are presented to assess the performance of our proposed multi-objective robust resource allocation scheme. The simulation settings for our simulation are as follows. It is assumed that there are $K=3$ DL users, $M=2$ eavesdroppers, $N_{E}=2, N_{U}=3$, $N_{D}=2$, and $\varsigma=10^{-6}$. The small scale fading of the UL channel, DL channels, CCI channels, and eavesdropping channels are modeled as independent and identically distributed Rayleigh fading. All the users and eavesdroppers are assumed to be located randomly and uniformly in the range between the distance of 10 and $100 \mathrm{~m}$ away from the transmitter. The SI channel is modeled as the Rician fading channel with Rician factor $5 \mathrm{~dB}$. In regards to the channel uncertainties, we define the channel estimate error ratios as: $\alpha_{L, m}=\frac{\varepsilon_{L, m}}{\sqrt{E\left\{\left\|\overline{\mathbf{L}}_{m}\right\|_{F}^{2}\right\}}}, \alpha_{E, m}=\frac{\varepsilon_{E, m}}{\sqrt{E\left\{\left\|\overline{\mathbf{E}}_{m}\right\|_{F}^{2}\right\}}}, \forall m$, and $\alpha_{F, m}=\frac{\varepsilon_{F, m}}{\sqrt{E\left\{\left\|\overline{\mathbf{F}}_{m}\right\|_{F}^{2}\right\}}}, \forall k$. All simulation results were achieved by an average of 1000 channel realizations. In our simulation, the feasibility of the given parameters can be ensured in this way. Taking problem P1 in (12) as an example, the secrecy rates $\bar{R}^{U L-S}$ in constraint $C 1$ and $\bar{R}_{k}^{D L-S}$ in constraint $\mathrm{C} 2$ will be given first. Then, after many channel realizations, the value of $P_{\max }^{D L}$ can be determined by checking the feasibility of problem P1 without constraint C3. At the same time, the maximum allowable transmit power $P_{\max }^{U L}$ can also be determined by the obtained maximum objective value of problem P1. The infeasible case will be picked and assigned to null once the cvx status is not "Solved" or cvx optval is "Inf.' The infeasible case should not be occurred frequently among the 1000 channel realizations. If it happens, we need to increase the value of the maximum allowable transmit power until it occurs rarely.

Figure 1 depicts the trade-off between UL and DL transmit powers with $P_{\max }^{\mathrm{UL}}=5 \mathrm{~dB}$ and $P_{\max }^{\mathrm{DL}}=10 \mathrm{~dB}$. The UL user and $k$ th DL user minimum required secrecy rate are 


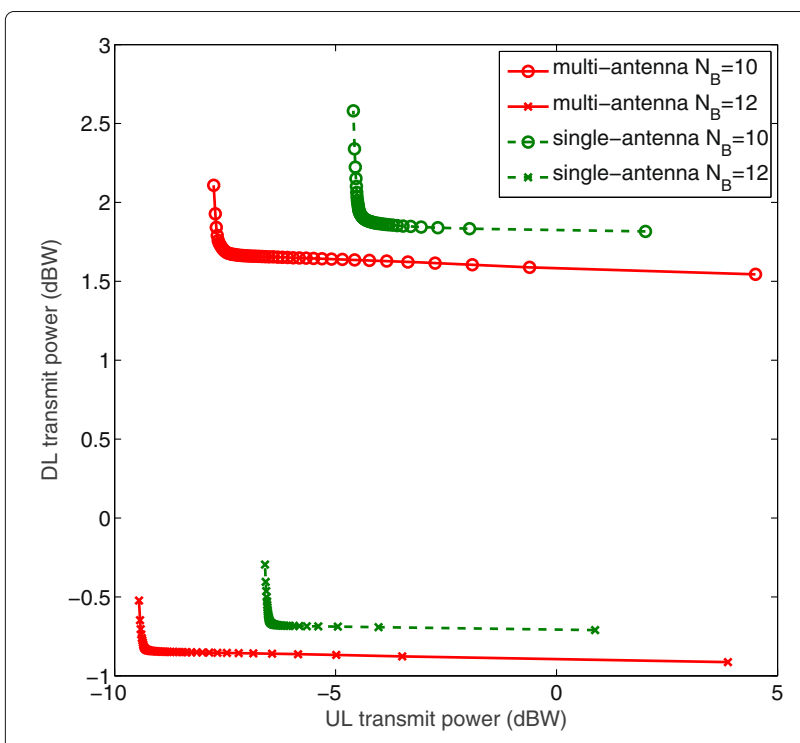

Fig. 1 Trade-off region between UL and DL transmit powers for different number of transmit and receive antennas

$\bar{R}^{\mathrm{UL}-\mathrm{S}}=1.5 \mathrm{bps} / \mathrm{Hz}$ and $\bar{R}^{\mathrm{DL}-\mathrm{S}}=2.5 \mathrm{bps} / \mathrm{Hz}$, respectively. The channel estimate error ratios are set as $\alpha_{L, m}=\alpha_{E, m}=$ $\alpha_{F, k}=0.05, \forall m, k$. The boundary points of the trade-off region are obtained by solving problem (P3) under different weight values $0 \leq \lambda_{j} \leq 1, j=1,2$ with fixed step size 0.02 .

For the purpose of a comprehensive performance comparison, four scenarios where each of the users and eavesdroppers is equipped with multi-antenna and $N_{B}=10$, where each of the users and eavesdroppers is equipped with multi-antenna and $N_{B}=12$, where each of the users and eavesdroppers is equipped with single antenna and $N_{B}=10$ and where each of the users and eavesdroppers is equipped with single antenna and $N_{B}=12$ are considered in Fig. 1. From this figure, we can see that, under the case the same antenna equipped at the transmitter, an obvious savings in transmit power for UL user and DL transmitter can be achieved when the UL, DL users, and eavesdroppers have multiple antennas compared with the single-antenna cases. Particularly, the requirement of UL transmit power declined more significant than the DL transmit power. This is due to the fact that the change of the antenna number results in a direct influence on UL user while it is indirect on the transmitter. The direct and apparent impact which leads to obvious power saving on the transmitter can be achieved with the increase of the number $N_{B}$ while the antennas of the other nodes keep unchanged. An interesting phenomenon what we can learn from this figure is that, from the first and the last curves listed in the legend, the multi-antenna case with $N_{B}=10$ consumes much more DL transmit power but less UL transmit power than the single-antenna case with
$N_{B}=12$. It further reveals the advantage of more antennas allocated for the communication nodes, especially for the users.

Figure 2 shows the convergence performance of our proposed algorithm. The initial values of $\left\{\mathbf{W}_{k 0}\right\}_{k=1}^{K}, \mathbf{V}_{0}$ and $\boldsymbol{\Sigma}_{0}$ are all set to be $\mathbf{0}$, the channel uncertainties and minimum required secrecy rates are the same as in Fig. 1, multi-antenna case is considered with $N_{B}=10$. The black curves marked with $\mathrm{x}$ represent the DL transmit power (corresponding to problem P2) while the blue curves marked with circle represent UL transmit power (corresponding to problem P1). Ten random channel realizations are depicted in Fig. 2. From this figure, we can see that our proposed algorithm converges to a stable value after a finite number of iteration. This is due to the fact that the problem defined in $(\widetilde{\mathrm{P} 3})$ is convex, the optimal transmit covariance matrix $\mathbf{W}_{k}^{*}, \mathbf{V}^{*}$, and $\Sigma^{*}$ are obtained by solving problem ( $\widetilde{\mathrm{P}} 3)$ for the given $\mathbf{W}_{k 0}, \mathbf{V}_{0}$, and $\Sigma_{0}$. At each iteration, $\mathbf{W}_{k 0}, \mathbf{V}_{0}$, and $\Sigma_{0}$ will be updated from the optimal $\mathbf{W}_{k}^{*}, \mathbf{V}^{*}$, and $\Sigma^{*}$ of the previous iteration. Hence, $\mathbf{W}_{k 0}, \mathbf{V}_{0}$, and $\Sigma_{0}$ are always the feasible solution of the next iteration, and the optimal solution $\mathbf{W}_{k}^{*}, \mathbf{V}^{*}$, and $\Sigma^{*}$ obtained for the given $\mathbf{W}_{k 0}, \mathbf{V}_{0}$, and $\Sigma_{0}$ will consume less than or equal to the transmission power of the previous iteration. Then, the required transmission power will monotonically non-increasing at each iteration. Moreover, since the transmission power is lower bounded below for a required secrecy rate, this algorithm will converge to a solution. Moreover, the convergence step of two problems appears to be consistent.

Figure 3 shows the average power consumption of UL user and DL transmitter versus the channel estimate error ratios. The antennas of the DL transmitter is set to be $N_{B}=10$. Three cases, non-robust, our proposed robust

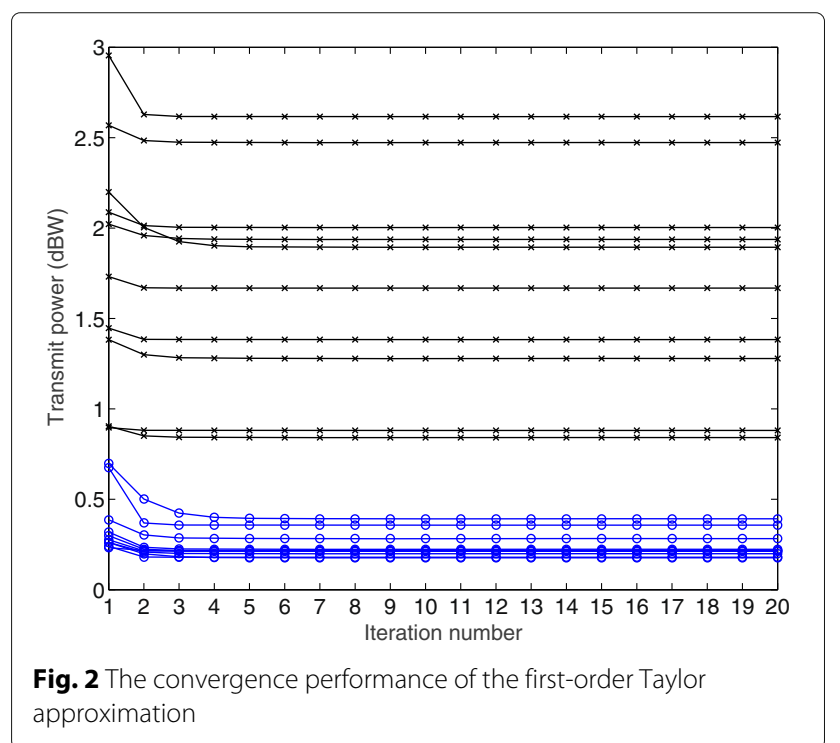




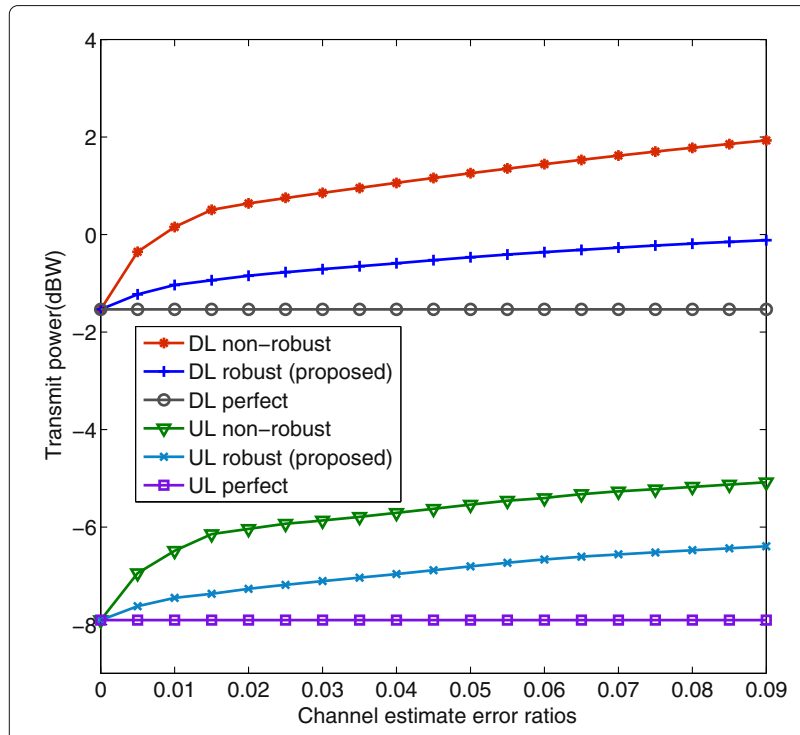

Fig. 3 Average power consumption versus the channel estimate error ratios

and the perfect CSI known at the DL transmitter, are depicted in Fig. 3. The information of channels $\mathbf{L}_{m}, \mathbf{E}_{m}$, and $\mathbf{F}_{k}$ known at the base station are actually imperfect. Physically, the non-robust case is that the researchers mistakenly believe that the CSIs known by the base station are perfect and do not take any additional actions to against the disadvantage caused by channel uncertainties. To reflect the actual situation, for the non-robust case, the channel estimate errors (each of them comes from the channel estimate error ratio multiplied by channel estimation) are added in the estimated channels. The optimization problem for the non-robust case is nonconvex due to the difference between two logarithmic functions in constraints $\mathrm{C} 1$ and $\mathrm{C} 2$, which can be tackled by using the similar methods adopted in Section 3. From this figure, we can see that, to meet the QoS requirement of secrecy rates of UL and DL users, the transmitter power of both UL user and DL transmitter increases with the channel uncertainties for the non-robust and robust cases. For the perfect CSI case, the consumed power remains unchanged and lower than the other two cases. In regard to our proposed robust scheme, a significant power saving is fulfilled compared with the non-robust scheme.

Figure 4 shows the trade-off between UL and DL transmit power for our proposed robust and the existing non-robust schemes. $(0.5,1.5)$ and $(2.5,3.5)$ denote the required secrecy rate pairs while $(0.5,1.5)$ denotes $\bar{R}^{U L-S}=0.5 \mathrm{bps} / \mathrm{Hz}$ and $\bar{R}^{D L-S}=1.5 \mathrm{bps} / \mathrm{Hz},(2.5,3.5)$ denotes $\bar{R}^{U L-S}=2.5 \mathrm{bps} / \mathrm{Hz}$ and $\bar{R}^{D L-S}=3.5 \mathrm{bps} / \mathrm{Hz}$. The number of the antennas of the base station is $N_{B}=12$. The maximum allowable transmit power, the channel estimate error ratios and the step size are the same as that

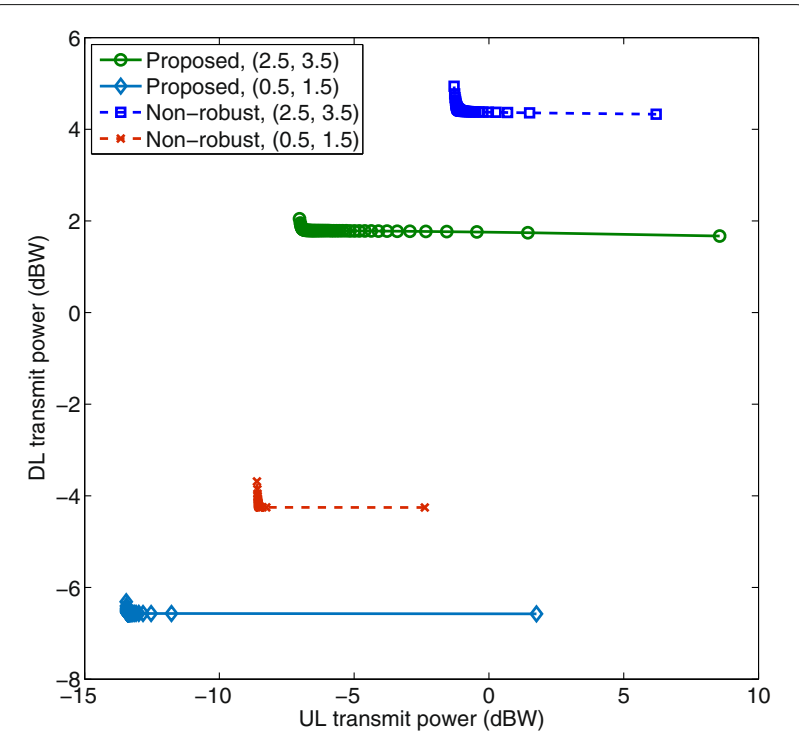

Fig. 4 Trade-off region between UL and DL transmit powers for different schemes

given in Fig. 1. From this figure, we can see that the transmit power of the UL user and the base station increase significantly with the required secrecy rates. Our proposed scheme consumes less power at the transmitters than the non-robust scheme under the same required secrecy rate pair. Moreover, by comparing the curve "Proposed, (2.5, $3.5)$ " in Fig. 4 with the curve "multi-antenna $N_{B}=10$ " in Fig. 1, we find that both of them achieve nearly the same UL and DL transmit power trade-off region. This means that the higher secrecy rate can be obtained by increasing the degrees of freedom with more transmit antennas instead of the transmit power.

Figure 5 shows the number of the infeasible case occurred versus the UL transmit power for five different DL transmit power, given $\bar{R}^{\mathrm{UL}-\mathrm{S}}=1.5 \mathrm{bps} / \mathrm{Hz}$ and $\bar{R}^{\mathrm{DL}-\mathrm{S}}=$ $2.5 \mathrm{bps} / \mathrm{Hz}$. From this figure, we can see that the probability of the infeasible case does not exceed one percent at the low transmit power region, which is absolutely a small probability event and can be accepted. Moreover, the frequency of this event decreases with transmit power and eventually tends to zero at the high transmit power region.

\section{Conclusions}

In this paper, we have studied the robust resource allocation problem for the multi-user full-duplex (FD) multipleinput multiple-output (MIMO) communication systems. Considering the existence of passive eavesdroppers, the QoS requirement of secrecy communication for both UL and DL users were incorporated in our considered problem. A multi-objective optimization framework was proposed to minimize the UL and DL transmission power simultaneously. The weighted Tchebycheff coupled 


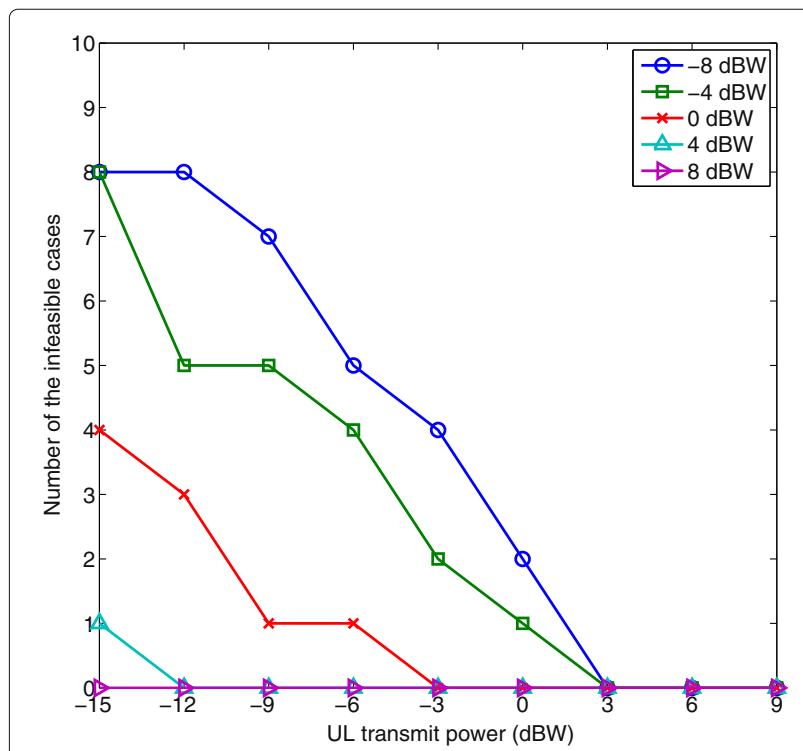

Fig. 5 The infeasible case occurs among 1000 channel realizations for different transmit power

with the Taylor series expansion and the S-procedure approaches were proposed in handing the formulated non-convex optimization problem optimally. Simulation results not only demonstrated an interesting trade-off between the considered conflicting objectives, but also showed the efficiency of our proposed robust resource allocation designs.

\section{Acknowledgements}

The work was partially supported by Swedish Research Links [No.348-2008-6212], the National Natural Science Foundation of China [61571241], Industry-university-research prospective joint project of Jiangsu Province [BY2014014], and Major projects of Jiangsu Province university natural science research [15KJA510002].

\section{Authors' contributions}

Both authors contributed extensively to the work presented in this paper. MZ designed the proposed algorithm, performed the experiments, and wrote and revised the paper. DZ supervised the design of the algorithm and the performance evaluation, and revised the paper. Both authors read and approved the final manuscript.

\section{Competing interests}

The authors declare that they have no competing interests.

\section{Publisher's Note}

Springer Nature remains neutral with regard to jurisdictional claims in published maps and institutional affiliations.

\section{Author details \\ ${ }^{1}$ Internet of Things, Nanjing University of Posts and Telecommunications, Nanjing 210003, China. ${ }^{2}$ Information Technical Department, Wenzhou Vocational Technical College, Wenzhou 325000, China.}

Received: 1 January 2017 Accepted: 24 October 2017

Published online: 14 November 2017

\section{References}

1. P Yeoh, M Elkashlan, N Yang, D da Costa, T Duong, Unified analysis of transmit antenna selection in MIMO multirelay networks. IEEE Trans. Veh. Technol. 62(2), 933-939 (2013)
2. J Park, B Clerckx, Joint wireless information and energy transfer in a two-user MIMO interference channel. IEEE Trans. Wirel. Commun. 12(8), 4210-4221 (2013)

3. X Chen, Z Zhang, H-H Chen, H Zhang, Enhancing wireless information and power transfer by exploiting multi-antenna techniques. IEEE Commun. Magazine. 53(4), 133-141 (2015)

4. A Khisti, GW Wornell, Secure transmission with multiple antennas-Part II: the MIMOME wiretap Cchannel. IEEE Trans. Inf. Theory. 56(11), 5515-5532 (2010)

5. DWK Ng, ES LO, R Schober, Dynamic resource allocation in MIMO-OFDMA systems with full-duplex and hybrid relaying. IEEE Trans. Commun. 60(5), 1291-1304 (2012)

6. H Suraweera, I Krikidis, G Zheng, C Yuen, P Smith, Low-complexity end-to-end performance optimization in MIMO full-duplex relay systems. IEEE Trans. Wirel. Commun. 13(2), 913-927 (2014)

7. D Nguyen, L-N Tran, P Pirinen, M Latva-aho, On the spectral efficiency of full-duplex small cell wireless systems. IEEE Trans. Wirel. Commun. 13(9), 4896-4910 (2014)

8. HQ Ngo, H Suraweera, M Matthaiou, E Larsson, Multipair full-duplex relaying with massive arrays and linear processing. IEEE J. Select. Areas Commun. 32(9), 1721-1737 (2014)

9. Y Sun, DWK Ng, J Zhu, R Schober, Multi-objective optimization for robust power efficient and secure full-duplex wireless communication systems. IEEE Trans. Wirel. Commun. 15(8), 5511-5526 (2016)

10. S Leng, DWK Ng, N Zlatanov, R Schober, in 2016 IEEE International Conference on Communications (ICC), Kuala Lumpur. Multi-objective resource allocation in full-duplex SWIPT systems, (2016), pp. 1-7. http://ieeexplore.ieee.org/document/7510760/

11. DWK Ng, ES Lo, R Schober, Multi-objective resource allocation for secure communication in cognitive radio networks with wireless information and power transfer. IEEE Trans. Veh. Technol. 65(5), 3166-3184 (2016)

12. $Y$ Zeng, $R$ Zhang, Full-duplex wireless-powered relay with self-energy recycling. IEEE Wirel. Commun. Lett. 4(2), 201-204 (2015)

13. B Day, A Margetts, D Bliss, P Schniter, Full-duplex MIMO relaying: achievable rates under limited dynamic range. IEEE J. Select. Areas Commun. 30(8), 1541-1553 (2012)

14. Y Liang, G Kramer, HV Poor, S Shamai, in 2008 IEEE 19th International Symposium on Personal, Indoor and Mobile Radio Communications, Cannes. Recent results on compound wire-tap channels, (2008), pp. 1-5. http:// ieeexplore.ieee.org/document/4699909/

15. Z Chu, K Cumanan, Z Ding, M Johnston, SY Le Goff, Secrecy rate optimizations for a MIMO secrecy channel with a cooperative jammer IEEE Trans. Veh. Technol. 64(5), 1833-1847 (2015)

16. W Wu, B Wang, Robust secrecy beamforming for wireless information and power transfer in multiuser MISO communication system. EURASIP J. Wirel. Commun. Netw. 2015(161) (2015)

17. Z Chu, Z Zhu, M Johnston, SY Le Goff, Simultaneous wireless information power transfer for MISO secrecy channel. IEEE Trans. Veh. Technol. 65(9), 6913-6925 (2016)

18. Z Chu, K Cumanan, Z Ding, M Johnston, S Le Goff, Robust outage secrecy rate optimizations for a MIMO secrecy channel. IEEE Wirel. Commun. Lett. 4(1), 86-89 (2015)

19. RT Marler, JS Arora, Survey of multi-objective optimization methods for engineering. Struct. Multidiscip. Optim. 26, 369-395 (2004)

20. W Wu, B Wang, Efficient Transmission solutions for MIMO wiretap channels with SWIPT. IEEE Commun. Lett. 19(9), 1548-1551 (2015)

21. GH Golub, CF van Loan, Matrix computation, 2nd edn. (John Hopkins University Press, 1989)

22. K Cumanan, Z Ding, B Sharif, GY Tian, KK Leung, Secrecy rate optimizations for a MIMO secrecy channel with a multiple-antenna eavesdropper. IEEE Trans. Veh. Technol. 63(4), 1678-1690 (2014)

23. S Boyd, L Vandenberghe, Convex optimization. (Cambridge University Press, 2004)

24. Z-Q Luo, JF Sturm, S Zhang, Multivariate nonnegative quadratic mappings. SIAM J. Optimiz. 14, 1140-1162 (2004)

25. J Sturm, Using SeDuMi 1.02, a MATLAB toolbox for optimization over symmetric cones. Optim. Methods Softw. 11, 625-653 (1999). (webpage and software). http://sedumi.ie.lehigh.edu/?page_id=58. SeDuMi 1.3 (2003) 